\title{
Intoxicación por litio. A propósito de un caso de disfunción multiorgánica
}

\begin{abstract}
Helena Sofia Santos-Gonçalves', Andre Terras Alexandre², Ana Filipa Rebelo
'Departamento de Medicina Interna. Hospital de Vila Real. Centro Hospitalar de Trás os Montes e Alto Douro. Vila Real. Portugal

${ }^{2}$ Departamento de Neumología. Hospital de Vila Real. Centro Hospitalar de Trás os Montes e Alto Douro. Vila Real. Portugal
\end{abstract}

Recibido: 16/07/2018

Aceptado: 30/10/2018

En línea: 31/12/2018

Citar como: Santos-Gonçalves HS, Alexandre AT, Rebelo AF. Intoxicación por litio. A propósito de un caso de disfunción multiorgánica. Rev Esp Casos Clin Med Intern (RECCMI). 2018 (Dic); 3(3): 125-126. doi: 10.32818/reccmi.a3n3a8.

Autor para correspondencia: Helena Sofia Santos-Gonçalvez. helenasantosgoncalves@gmail.com

\begin{tabular}{l} 
Palabras clave \\
\hline$\triangleright$ Litio \\
$\triangleright$ Intoxicación \\
$\triangleright$ Hipotiroidismo \\
$\triangleright$ Miocardiopatía crónica
\end{tabular}

\begin{tabular}{l}
\hline Keywords \\
\hline$\triangleright$ Lithium \\
$\triangleright$ Poisoning \\
$\triangleright$ Hypothyroidism \\
$\triangleright$ Chronic cardiomyopathy \\
\hline
\end{tabular}

\section{Resumen}

El litio es un fármaco ampliamente utilizado y tiene un rango terapéutico muy estrecho debido a su potencial toxicidad. La intoxicación por litio puede afectar a diversos órganos y tener diferentes tipos de presentación. Presentamos un caso de toxicidad multiorgánica por litio, en un paciente bajo tratamiento crónico por trastorno bipolar.

Abstract
Lithium is a widely used drug which has a very narrow therapeutic range, because of its potential of toxicity. Lithium
poisoning can affect vary organs, and have different pardons of presentation. We present a multi-organ lithium toxi-
city case in a patient under chronic treatment with lithium for bipolar disease.

\section{Puntos destacados}

$\triangleright$ Se presenta el caso de un paciente con afectación cardíaca, tiroidea y renal por intoxicación por litio.

$\triangleright$ La marcha diagnóstico y la estrategia terapéutica en este caso clínico demuestran la dificultad e importancia del diagnóstico precoz de intoxicación por litio.

\section{Introducción}

El litio es un medicamento ampliamente usado para el tratamiento de enfermedades psiquiátricas. Además de ser un fármaco eficaz, su uso debe ser sometido a un control estricto debido a su potencial toxicidad. La intoxicación por litio puede presentarse de tres formas diferentes: aguda, en personas que toman una sobredosis de comprimidos; crónica, cuando la ingesta supera su eliminación durante varias semanas; y agudo-cronica, cuando un paciente con intoxicación crónica toma dosis adicionales ${ }^{1-3}$.

\section{Caso clínico}

Hombre de 38 años, antecedentes de trastorno bipolar desde los 16 años de edad, prediabetes y dislipidemia. Admitido en el Servicio de Urgencias por presentar debilidad, letargo, dolor en las piernas, polidipsia y poliuria de 5 días de duración. Su tratamiento habitual es carbonato de litio $400 \mathrm{mg} / 8 \mathrm{~h}$ en los últimos 7 años, clomipramina 75 mg/12 h, clorazepato dipotásico 15 mg/8 h, olanzapina $2,5 \mathrm{mg} / \mathrm{día}$, fenofibrato $275 \mathrm{mg} / \mathrm{d}$ á, rosuvastatina $10 \mathrm{mg} / \mathrm{día}$, metformina $1 \mathrm{~g} / \mathrm{día}$. El paciente acudía de forma irregular a consulta y no se conocían los niveles previos de litio.

En el Servicio de Urgencias, se encuentra despierto, buena colaboración, letárgico, deshidratado, pálido, apirético, normotenso, con ligeros edemas periféricos. Analíticamente destaca la presencia de anemia normocítica normocrómica (Hgb 10,61 g/dl), glucosa 426 mg/dl, urea/creatinina (U/C) 73/2,1 mg/dl, sodio $119 \mathrm{mEq} / \mathrm{l}$, potasio 7,0 mEq/l, calcio 1,28 mmol//, osmolaridad sanguínea $266 \mathrm{mOsm} / \mathrm{kg}$, troponina T 0,145 ng/ml, mioglobina 66,1 ng/ml, creatina-cinasa MB 3,69 ng/ml, pro-BNP 167,6 pg/ml, HbA1c 14,3\%, colesterol total 476 mg/ dl, colesterol HDL 58 mg/dl, colesterol LDL 120 mg/dl, triglicéridos $1.661 \mathrm{mg} /$ dl, litio 3,00 mmol/l (nivel tóxico > 2), hormona estimulante del tiroides (TSH) $67,5 \mathrm{mUI} / \mathrm{l}$ (rango terapéutico: 0,27-4,20 mUI). El análisis de orina evidencia 0,5 mg/dl de proteínas, $>1 \mathrm{mg} / \mathrm{dl}$ de glucosa, sin otras alteraciones. El electrocardiograma muestra ondas Q y supra-ST en III y aVF, y en el ecocardiograma se observa una reducción de la función sistólica, acinesia del ápex y de los segmentos distales.

El paciente ingresa en la Unidad de Medicina Interna con el diagnóstico de intoxicación por litio, con miocardiopatía, hipotiroidismo e insuficiencia renal aguda. El estudio adicional revela hormona vasopresina y osmolaridad urinaria normal. La ecografía abdominal evidencia hepatomegalia con esteatosis difusa 
y riñones hiperecogénicos. La ecografía tiroidea muestra una glándula tiroides de tamaño normal con textura difusamente hipoecogénica, y los anticuerpos tiroideos son negativos. El estudio de autoinmunidad es negativo, y la recolección de orina de 24 horas muestra microalbuminuria 251 mg/día, proteínas 497 mg/día y sodio 149 mEq/día. Se realiza resonancia magnética cardíaca que demuestra disfunción sistólica ventricular izquierda ligera, FSVE 48\%, sin signos de fibrosis/cicatriz del miocardio. Durante el ingreso en el hospital, el paciente se recupera con terapia de soporte, levotiroxina e insulina. Al alta hospitalaria, es tratado con ácido acetilsalicílico, carvedilol, lisinopril, fibrato, gliclazida, vildagliptina, insulina glargina, levotiroxina, ácido valproico, olanzapina, ácido fólico hierro, epoetina. Pasados 9 meses, se repitió ecocardiograma que mostró recuperación completa de la función sistólica, manteniendo todavía disfunción renal.

\section{Discusión}

El principal problema del uso de litio es su estrecho índice terapéutico, porque los niveles ligeramente superiores al rango terapéutico pueden inducir síntomas de intoxicación ${ }^{3}$. La capacidad del litio para inducir toxicidad depende de varios factores: dosis, duración del tratamiento, niveles séricos, interacción de fármacos y factores del paciente, como la deshidratación. Diversos órganos y sistemas pueden verse afectados por la intoxicación con litio, como los sistemas nervioso, cardiovascular, gastrointestinal, tiroideo y renal ${ }^{3}$.

La disfunción tiroidea es una complicación bien conocida del litio, y puede presentarse como bocio con o sin hipertiroidismo o hipotiroidismo, siendo el último la forma más común², . En el hipotiroidismo inducido por litio, los síntomas del paciente no son diferentes, pero pueden enmascararse debido a su similitud con los síntomas de depresión que estos pacientes suelen padecer Sexo femenino, edad avanzada, anticuerpos tiroideos positivos e historia familiar de disfunción tiroidea, son factores de riesgo conocidos para desarrollar disfunción tiroidea cuando se toma litio ${ }^{4}$. Nuestro paciente no presentaba ninguno de estos factores de riesgo, pero la ecografía tiroidea fue característica de tiroiditis y la ausencia de anticuerpos excluyó la causa autoinmune, lo que nos permite creer que el litio fue la causa del hipotiroidismo severo. Esta disfunción también explica la hipertrigliceridemia severa y la esteatosis hepática que el paciente presentó al ingreso.

En relación con la disfunción cardíaca inducida por el litio, las manifestaciones más descritas en la literatura son alteraciones electrocardiográficas y del ritmo, contrariamente a la disfunción del miocardio, que no está bien establecida ${ }^{5}$. Sin embargo, algunos informes de casos han reforzado el hecho de que el litio en sí mismo puede ser una causa de miocardiopatía ${ }^{6,7}$. En nuestro caso, el paciente tenía factores que podrían ser una causa de disfunción cardíaca, como factores de riesgo cardiovascular e hipotiroidismo. La ausencia de fibrosis/cicatrices miocárdicas en los exámenes de imagen excluyó la isquemia como causa. Con respecto al hipotiroidismo aunque es una causa conocida de disfunción cardíaca ${ }^{8}$, el hecho de que la intoxicación con litio haya sido la causa del mismo hipotiroidismo nos permite creer que el litio actuó como factor tóxico miocárdico antes que el fallo del tiroides. De algun modo, la intoxicación con litio y el hipotiroidismo promovieron sinérgicamente la disfunción cardíaca. La recuperación rápida de la función cardíaca después de la suspensión del litio también refuerza la posibilidad de que el litio sea una causa de insuficiencia cardíaca.

El riñón también es un órgano que frecuentemente está involucrado en la toxicidad del litio. Algunos estudios revelaron que las lesiones renales más frecuentes son la nefropatía tubulointersticial y la diabetes insípida nefrogénica $(\mathrm{NDI})^{2,3}$. En nuestro caso, no hubo evidencia de NDI, por lo que creemos que el paciente desarrolló una necrosis tubular aguda en el contexto de intoxicación crónica con litio, exacerbada por deshidratación, hipotiroidismo y diabetes descompensada.

La intoxicación crónica por litio es una situación clínica muy peligrosa, no sólo por su capacidad para promover lesiones en varios órganos, sino también porque puede agravarse por situaciones que aumentan los niveles de litio como sobredosis voluntarias o la situación de deshidratación ${ }^{3}$. Teniendo en cuenta el cuadro clínico descrito, y el hecho de que el paciente no tenía visitas médicas regulares, pensamos que el paciente sufrió una intoxicación crónica por litio no detectada precozmente asociada a disfunción de varios órganos y exacerbada por la diabetes sintomática y el hipotiroidismo severo. Por lo que respecta a la persistencia de la insuficiencia renal, consideramos que está relacionada con su cumplimiento terapéutico, que sigue siendo irregular, y que también se puede explicar por el hecho de que los pacientes que no recuperan la función renal después de la retirada del litio tienen una mayor probabilidad de evolución a un estadio de insuficiencia renal terminal².

\section{Bibliografía}

1. Altschul E, Dougherty R, Gaikwad R, Nguyen V, Schwimmer J, Merker E, Mandel S. Lithium toxicity: a review of pathophysiology, treatment, and prognosis. Practical neurology. 2016 March: 42-45.

2. Gitlin M. Lithium side effects and toxicity: prevalence and management strategies. Int J Bipolar Disord. 2016; 4(1): 27.

3. Baird-Gunning J, Lea-Henry T, Hoegberg LCG, Gosselin S, Roberts DM. Lithium poisoning. J Intensive Care Med. 2017 May; 32(4): 249-263. doi: 10.1177/0885066616651582.

4. Kibirige D, Luzinda K, Ssekitoleko R. Spectrum of lithium induced thyroid abnormalities: a current perspective. Thyroid Res. 2013 Feb 7; 6(1): 3. doi: 10.1186/1756-6614-6-3.

5. Brady HR, Horgan JH. Lithium and the heart. Unanswered questions. Chest. 1988; 93(1): 166-169.

6. Aichhorn W, Huber R, Stuppaeck C, Whitworth AB. Cardiomyopathy after long-term treatment with lithium -more than a coincidence? J Psychopharmacol. 2006; 20(4): 589-591.

7. Anantha Narayanan M, Mahfood Haddad T, Bansal O, Baskaran J, Azzouz MS, Akinapelli A, et al. Acute cardiomyopathy precipitated by lithium: is there a direct toxic effect on cardiac myocytes? Am J Emerg Med. 2015 Sep; 33(9): 1330.e1-5. doi: 10.1016/j.ajem.2015.03.023.

8. Grais IM, Sowers JR. Thyroid and the heart. Am J Med. 2014 Aug; 127(8): 691698. doi: 10.1016/j.amjmed.2014.03.009. 